\title{
Leveraging the new with the old: providing a framework for the integration of historic microarray studies with next generation sequencing
}

\author{
Michael A Bauer, Shweta S Chavan, Erich A Peterson, Christoph J Heuck, Donald J Johann Jr*
}

From 11th Annual MCBIOS Conference

Stillwater, OK, USA. 6-8 March 2014

\begin{abstract}
Next Generation Sequencing (NGS) methods are rapidly providing remarkable advances in our ability to study the molecular profiles of human cancers. However, the scientific discovery offered by NGS also includes challenges concerning the interpretation of large and non-trivial experimental results. This task is potentially further complicated when a multitude of molecular profiling modalities are available, with the goal of a more integrative and comprehensive analysis of the cancer biology.

Microarray transcriptome analyses have resulted in important advances in both the scientific and clinical domains of biomedicine. Importantly, as technology advances, it is critical to leverage what has been gained from historic approaches (e.g., microarrays) with new approaches (NGS). In this regard, necessity dictated a need to utilize and leverage the many years of historical microarray data with new NGS approaches. This is especially important since NGS approaches are now entering clinical medicine. For instance, NGS-based comprehensive analysis of certain cancers has already helped to uncover specific mutations that contribute to the malignant process, identify new therapeutic targets, and improve opportunities for choosing the best treatment for an individual patient. A suite of custom software tools have been developed to rapidly integrate, explore, discover and validate molecular profiling data from the NGS modalities of Whole Exome Sequencing (WES) and RNA-seq with each other, as well as with historical microarray and salient clinical datasets. Importantly, our approach is independent of any particular type of NGS suite(s) or cancer types. This novel bioinformatic framework is now assisting with the scientific and clinical management of patients with multiple myeloma.
\end{abstract}

\section{Background}

Next generation sequencing (NGS) is a new frontier in cancer and biomedical research, and these approaches are rapidly becoming the preferred method for human disease-based analysis due to vastly improved genome coverage and resolution [1]. Recently, the FDA granted marketing authorization for the first high-throughput NGS system (Illumina MySeqDx) for the purpose of clinical test development [2]. This FDA decision is

\footnotetext{
* Correspondence: don.johann@gmail.com

Myeloma Institute for Research and Therapy, University of Arkansas for Medical Sciences, Little Rock, AR, USA
}

(c) 2014 Bauer et al licensee BioMed Central Ltd. This is an Open Access article distributed under the terms of the Creative Commons Attribution License (http://creativecommons.org/licenses/by/4.0), which permits unrestricted use, distribution, and reproduction in any medium, provided the original work is properly cited. The Creative Commons Public Domain Dedication waiver (http:// creativecommons.org/publicdomain/zero/1.0/) applies to the data made available in this article, unless otherwise stated. enabling a new understanding of cancer. For instance, recent work from The Cancer Genome Atlas (TCGA) has shown that a particular cancer tissue of origin may be less relevant to therapeutic response and prognosis than the collection of causative mutations [3]. Thus, for cancer scenarios where the standard-of-care options are poor (e.g., drug resistance, metastasis), drug assignments based on the mutational landscape of a patient's tumor can sometimes provide significant benefit, and are a very active research topic in clinical trials and translational medicine [4]. However, NGS also brings new demands regarding the size and complexity of the 
associated data sets. These "big data" challenges are further magnified when multiple NGS modalities are utilized and there are needs or requirements to integrate this data with other molecular profiling techniques (e.g., microarrays).

NGS and related studies have already contributed significantly to the improved understanding of multiple myeloma (MM) [5-7]. Most recently there were 203 paired tumor/normal DNA samples analysed by either Whole Exome Sequencing (WES) or Whole Genome Sequencing (WGS) [8]. A principal finding was a very complex genetic landscape with extensive clonal heterogeneity that serves to limit scientific and clinical utility. In this case it appears DNA analysis is necessary but not sufficient. An approach to the genetic complexity and heterogeneity issue is to combine additional modalities, for example transcriptome data. By enhancing a DNA examination with transcriptome data, a multi-modality study of a particular patient's cancer is formed, yielding increased scientific/analytical rigor and potential insights. This is a primary aim of our methodology.

Microarrays have contributed significantly towards an improved understanding of MM and many other cancers, and there are large archives in the private and public domains. Thus, a familiarity with the analytic nature of transcriptome data from different modalities is important, especially regarding the explanatory abilities for cancer biology questions. To this end, a comparison of microarray vs. RNA-seq is provided in additional file 1[9]. In contrast to RNA-seq, microarray data is compressed and is largely a correlative science. RNA-seq requires less sample material, has base level resolution, a much larger dynamic range, is discovery-based for both novel isoforms and gene fusions, and can distinguish known splice isoforms.

For a cancer center or institute that has a focus on a particular cancer type, and who have a large volume of microarray data, the evolutionary shift in technology (i.e., microarray to NGS) can have disruptive effects on scientific and clinical workflows. Hence, there is a significant need to integrate and leverage historic molecular profiling studies (e.g., microarray) with new and existing NGS modalities. Additionally, the ability to capture, model, and successfully carry forward key aspects of a particular cancer biology learned from the legacy data and incorporate this knowledge into new and evolving software approaches is challenging, but needs to be carefully considered.

This study is a continuation of efforts to develop efficient software methodologies to allow for the incorporation and analysis of experimental data from a variety of molecular profiling modalities [10]. The current and historical work involving microarray technology to study the cancer biology of MM at the Myeloma Institute for Research and Therapy (MIRT), at the University of Arkansas for
Medical Sciences (UAMS), has resulted in over 19,000 microarray studies. This has included important insights into the cancer biology of MM, for example, the development of risk scores $[11,12]$ and a molecular classification [13]. A significant need exists to leverage this important work learned and gained from microarrays with the new and evolving NGS modalities.

Using a three tier approach the software has been redesigned, and extended to accommodate a variety of integration scenarios involving molecular profiling experimental data from i) microarrays, ii) RNA-seq, and iii) Whole Exome Sequencing (WES), as well as features to capture and model significant cancer biology items (e.g., prognostic gene groups and molecular classification). Figure 1 illustrates the information architecture of our NGS Association System. Importantly, our approach is independent of any particular type of transcriptome reconstruction, variant caller or annotator, quantitation tool, or file format. Input requirements simply consist of a tab delimited file with column one being a Gene ID (Ensembl, Entrez, or Genbank Accession) if known, column two being the gene symbol (if known), and column three a chromosome plus loci pair (if known). Additional columns are imported and associated in a set-based manner. We continue to integrate Affymetric probe set-IDs, and gene annotation information from an assortment of sources as previously reported, as well as employ a variety of strategies to maximize the integration, annotation, and association of biological information [10].

The novel aspects of our approach are the abilities that allow for a rapid integration, exploration, discovery and validation of large and complex experimental data from a wide variety of NGS and microarray molecular profiling modalities. For instance, the approach is useful for comparing the expression of genes of interest between RNA-seq and microarray. Other supported comparisons are: i) Whole Exome Sequencing (WES) vs. Microarray, ii) WES vs. RNA-seq, iii) WES vs. RNA-seq vs. Microarray (triple integration). The triple integration approach is illustrated in the paper since it is the most difficult. To our knowledge, there is no existing software suite available, commercial or open source, which provides the triple integration feature. These abilities enable a more informed view of the intricate details present in the complex cancer biology, especially in cases of extensive heterogeneity. Finally, our approach is independent of any particular cancer type. Multiple myeloma is used only as an illustrative example. Eventually, we plan to make our software available to the greater scientific community, possibly as open source.

\section{Methods}

Sample descriptions and library preparation

Bone marrow aspirates and one peripheral blood sample were collected at UAMS, MIRT from a single patient 


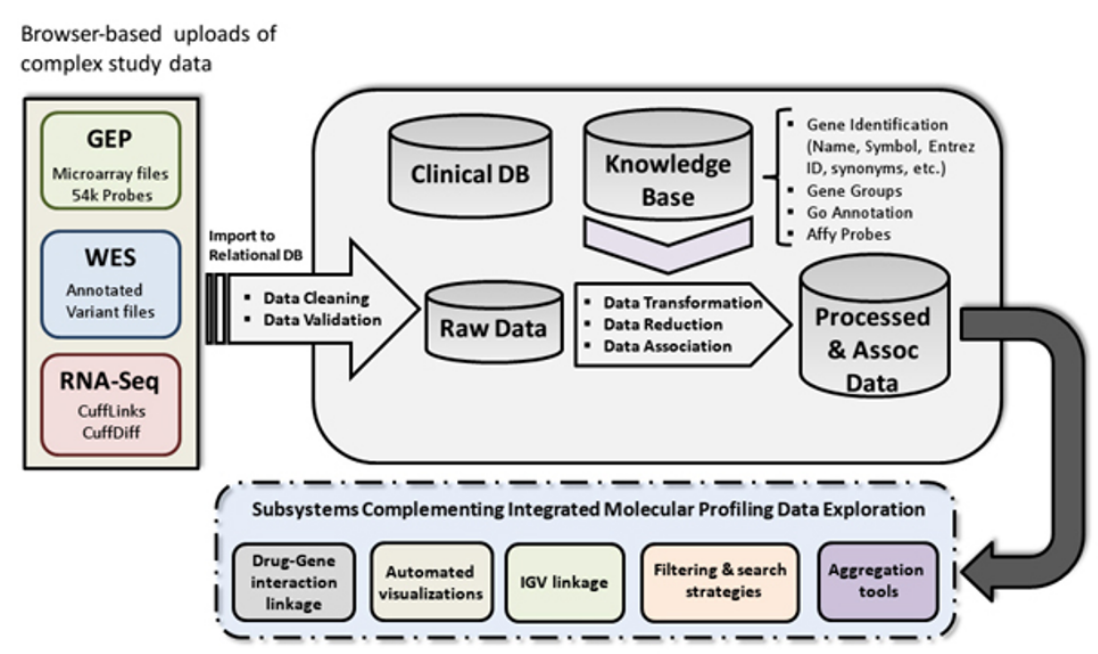

Figure 1 NGS Association System Information Architecture. The basis for integration of multiple molecular profiling modalities is illustrated. This includes processes for data transformation, reduction and association, as well as the direct interfacing to multiple custom and third party software tools and subsystems.

diagnosed with MM and three normal donors. The sample collection protocol was approved by the UAMS Institutional Review Board (IRB). Plasma cells from bone marrow aspirate samples were enriched by antiCD-138 immuno-magnetic bead selection in a central laboratory as previously described [14]. CD-138 is a marker for a malignant plasma cell, and for this sample the degree of CD-138 purification was $95 \%$. Germline material was obtained from the buffy coat following density gradient centrifugation of the peripheral blood sample. To ensure the absence of plasma cells, buffy coat material was also examined by flow cytometry. Microarray data files were obtained from the study archive section in our MM historical database and were processed as previously reported [12]. Note, GEO-based extraction using Bioconductor with GEOquery along with in-house developed data wrangling tools can also be used in workflows, provided the output is in a tab or comma separated value format in the order of : gene, probeID, value [15].

Whole exome capture libraries were constructed from $500 \mathrm{ng}$ of tumor and normal DNA after shearing, end repair, phosphorylation, and ligation to bar coded sequencing adaptors for a single patient. DNA was fragmented by the S220 focused-ultrasonicator (Covaris), using a standard protocol for a target bp of 300. DNA was size selected for lengths between $\sim 250$ - 330 bp and subjected to exonic hybrid capture using SureSelectXT Human All Exon V5 (Agilent). Libraries were enriched using 14 cycles of PCR. The library was run at a concentration of $8 \mathrm{pM}$. Samples were multiplexed and sequenced on an Illumina HiSeq 2500 using the rapid run mode (paired-end 101 bp reads) to an average depth of coverage of $100 x$, for tumor and normal respectively.

For the RNA-seq samples each Illumina mRNA-seq library was prepared using the TrueSeq mRNA kit v2. The starting sample material was $200 \mathrm{ng}$ of total RNA and fashioned according to the manufactures instructions. This was done for one patient with MM and three normal donors. Poly-A selection for mRNA isolation using streptavidin-coated magnetic beads, followed by thermal mRNA fragmentation per standard Illumina protocol, was used during the sample prep process. The fragmented mRNA was subjected to cDNA synthesis using reverse transcriptase according to the manufacturer's instructions. The cDNA was then converted into double stranded cDNA followed by an end repair process, and then ligated to Illumina paired end (PE) adaptors. Size selection was performed using AMPure XP beads (Beckman Coulter), generating cDNA libraries ranging in size from 300 to $350 \mathrm{bp}$ (base pairs). The library was enriched using 15 cycles of PCR and purified again by AMPure XP beads. The library was run at a concentration of $8 \mathrm{pM}$. The RNA-seq experiment was run on an Illumina HiSeq 2500 in the rapid run mode utilizing $101 \mathrm{bp}$ PE sequencing. In summary, the fragment length is $\sim 300-350 \mathrm{bp}$ and thus the inner-mate-distance is $\sim 98$ 148 bp since we are performing 101 bp PE sequencing.

\section{Whole exome sequencing (WES) data analysis and alignment}

Generation of FASTQ files was performed via CASAVA (http://support.illumina.com/sequencing/sequencing software/casava.html). Reads were analyzed and quality 
checked using FastQC (http://www.bioinformatics. babraham.ac.uk/projects/fastqc/). Based on the quality reports, it was decided to trim the last nine bases from each read, using Trimmomatic [16]. Paired-end reads were aligned to the human genome (GRCh37) by a hybrid approach that utilizes BWA [17] and Stampy [18]. Duplicate reads were removed using Picard tools (http://picard.sourceforge.net). Sequence recalibration and local realignment were performed using GATK [19]. Single nucleotide variant (SNV) and small insertions and deletions (InDels) calling was performed by Strelka [20]. SnpEff [21] was used to functionally annotate all variants.

\section{RNA-seq read alignment and transcriptome processing}

The RNA-seq data analysis utilized the Tuxedo suite. A standard pipeline protocol was utilized. Trimmomatic was utilized for preprocessing prior to alignment. RNA-seq reads were mapped using TopHat version 2.0.8 (http:// ccb.jhu.edu/software/tophat/index.html) against the human genome (GRCh37). Alignment utilized the following options: "-p 8 -G Ensembl.gtf”, where Ensembl.gtf contains the coding transcripts in GTF format. Cufflinks version 2.1.1 (http://cufflinks.cbcb.umd.edu/) was run for transcriptome reconstruction and quantitation for each generated BAM file with the following options: "-p 8 -g Ensembl.gtf", thus allowing for both annotation and novel transcript discovery. Normal donor assemblies were merged with Cuffmerge. Differential expression analysis between the MM sample and normal donor pool utilized Cuffdiff.

\section{System session management, data import, processing, and user interface \\ User profile management}

Additional file 2 illustrates the user profile management feature of our system. Subfigure A shows the User Profile tab. In this case, the "Login Name" is utilizing the UAMS domain account, which provides extra security and ease of use for this particular user. Various privileges for site administrator rights, as well as allowing for special debug modes, and IGV (Integrative Genomics Viewer) [22] integration are provided. Subfigure B displays the Gene groups tab that allows for the creation and modification of custom groups of genes for user driven search strategies.

\section{Upload of experimental dato}

Additional file 3 illustrates the experimental upload features. Provisions are provided to create new patient (or a symbol for a scientific study, e.g., mouse model, cell line, etc.) entries and this is shown in subfigure A. One or more experiments along with salient information can then be associated with a patient, etc. This is shown in subfigure B. Due to the large size of datasets, we have developed a scheduling strategy to accommodate data import and associative processing, which can be time intensive. Depending on the particular molecular profiling modality, one or several files may be uploaded to a server. Next, an import request is placed in a job queue. The import process will begin some of the basic data validation steps pertaining to the molecular profiling experimental data, and place it into a staging/raw set of database tables. Subsequently, the newly imported experimental data can now be subjected to a variety of transformations, associations, reductions, filtering operations, etc. The import task/process continually checks the queue, and processes the next available job, by either importing or transforming the user requested datasets. This is illustrated in subfigure $\mathbf{C}$.

\section{Data cleaning and validation}

Data integrity is paramount for accurate and reproducible science. In biomedicine and life sciences research, this can be a particularly challenging endeavour, given the arrival of big data from Next Generation Sequencing (NGS) experiments. The data is so massive that is becomes easy for errors to hide from detection. Thus, all files produced by the different NGS modalities were studied, and a specific parser has been designed for that file type. Some of the basic cleaning and validation steps include: giving the generic text data an explicit data type, range checks on all numbers, set membership checks on various symbol names/groups, etc. Incoming data must fit within certain expected ranges and formats before being placed in the appropriate database table. Aspects of these operations are shown in Figure 1.

Data transformation, reduction, and management

Once the data has successfully been imported into the database in its raw format, the next step is to perform the association and transformations to annotate and connect the different datasets. The sources of information used to construct our knowledge base, as well as the operation of the association algorithms, are modifications and enhancements of previously described methods [10]. Newly created ID association tables and software, acting as a switching center, allows for the conversion between different gene identifiers, and is shown in additional file 4. Conversion can be either direct or indirect depending on starting and target IDs. The ability to also convert between the different identifiers based on chromosome and loci has been added.

An executive console is illustrated in additional file 5 . This feature provides the ability to view experimental/study information on an aggregate basis, along with the particular modality, date loaded, number of rows (size) of various tables, etc. The ability to perform complex cascading delete operations on an entire study or just certain aspects is provided. 


\section{User interface}

Different experiments and modalities can be associated with a particular patient. On the patient view, available experiments may be viewed individually or in different combinations with other existing modalities, giving an integrated view. Additional file 6 displays an example of the Patient Experiments View, where for a particular patient, the available studies (RNA-seq, DNA, Microarray) for integration and viewing may be chosen. The user simply checks one or more of the left most boxes (next to Date), and then presses a "View" button. Additional file 7 contains a figure that shows results from the Cuffdiff Experiment for patient John Doe (Date 3/26/2014) following a simple selection and view. Subfigure A shows the initially loaded dataset and subfigure $\mathbf{B}$ shows a reduced dataset after applying a user defined custom filter consisting of five key genes.

Since users of the system will have different backgrounds and needs (e.g., clinical researcher vs. basic scientist), the ability to show a minimum or maximum number of columns/data elements has been provided. This is illustrated in the figures contained in additional files 8 and 9. Basic ergonomics are utilized regarding the display of grid columns, specifically column color schemes and their grid location, and are consistent regardless of the study, or studies loaded. For instance, the first three columns and last column are always: Entrez ID, Gene Symbol, Gene Name, and Member List, respectively. These are always colored grey in the referenced figures. Member List indicates the gene belongs to a certain user defined group(s), it is color coded but also has a "hover over" feature to reveal the group name (since gene groups may be shared among users). WES data/columns will appear next (if loaded) and these columns are always shaded green. RNA-seq columns then follow (not loaded in this example) and would be shaded light red. Finally, microarray columns appear last and are always shaded blue.

\section{Data visualization}

In addition to seeing the data in tabular formats, dynamically produced visualizations are created for different modalities to aid data explorations. Currently, these visualizations include simple bar and pie charts. These data-driven visualizations charts are created using NVD3 (http://nvd3.org) and D3 (http://d3js.org), and are demonstrated later in this study.

\section{IGV integration}

An integration option with IGV to promote the exploration and validation of the NGS data has been further developed. Using the IGV port interface the server sets up a socket connection to the users running a copy of IGV. Through this connection the server sends commands to IGV to load datasets for a particular NGS experiment. This automation allows the user to quickly jump to different locations within the genome, by a single click on the gene(s) of interest, in the web browser. Illustrated examples of this feature will appear in the results section.

\section{Systems integration testing}

A systems integration testing strategy was developed owing to the complexity of the NGS Association System software. Due to the utilization of third party tools and their custom developed interfaces, different software technologies (e.g., database stored procedures for fetching and set-based operations on large data sets, object oriented approaches for business logic, and a modern browser-based presentation system); along with extensive extraction, translate, and load (ETL) operations for the various molecular profiling modalities, as well as knowledge base construction and association operations, unit testing alone was not sufficient. Thus, a series of more elaborate testing scenarios are employed to exercise the entire NGS Association System in very deliberate ways, along with expected system actions and outputs.

\section{Results and discussion}

In this study, custom software methodologies have been further developed to allow for the rapid integration, exploration, discovery and validation of experimental data from a variety of molecular profiling modalities [10]. The current and historical work involving microarray technology to study the cancer biology of MM at MIRT has resulted in an extremely large microarray archive. Certainly, there is a need to leverage this important work with the new and evolving NGS modalities. Methods to incorporate cancer biology knowledge garnered from historic microarray studies, and to best aid with analysis approaches involving new NGS modalities, continue to be an active research topic. Importantly, for cancers known to be very genetically complex and heterogeneous (e.g., MM), DNA/WES is necessary but not sufficient, and an integrated multi-modality approach as presented in this study may aid with this dilemma.

The NGS Association system is independent of any particular cancer type. Molecular profiling results from a single patient with MM have been selected to illustrate our systems ability to rapidly find and analyse salient findings across multiple modalities. A diagram of the samples and molecular profiling studies used in the various analyses are presented in Figure 2. Although the outcomes for MM patients have considerably improved, those who are refractory to, or relapse following therapy with an IMiD and/or proteasome inhibitor have a poor prognosis [23]. NGS sequencing of MM patients have revealed multiple targetable mutations, including KRAS $[5,8]$.

Figure 3 shows the system rendered results of the Cuffdiff dataset that was searched using two genes having cancer biology significance in many cancers including MM (KRAS and TP53). The Cuffdiff Chart in subfigure $\mathbf{A}$ was 


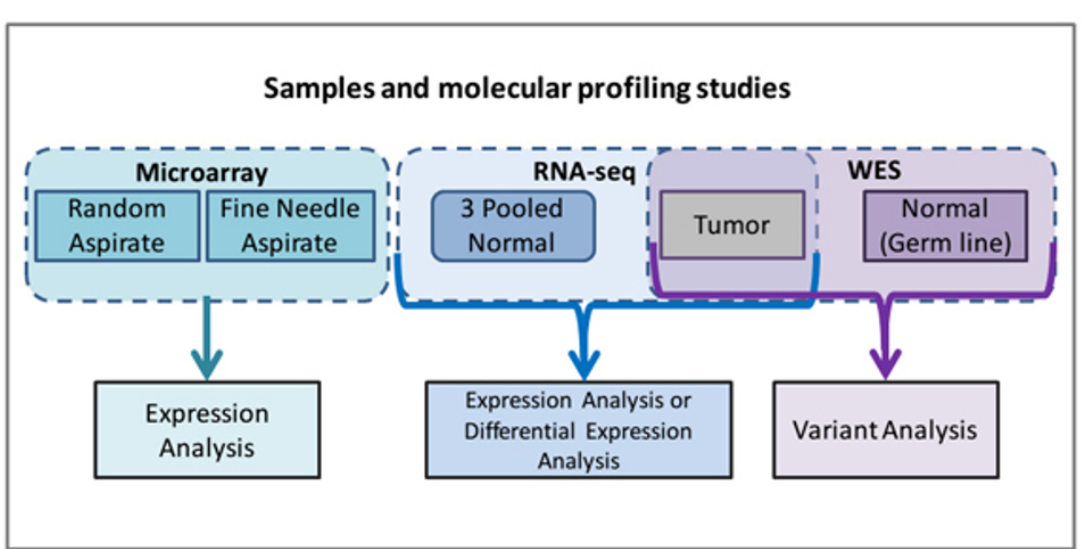

Figure 2 Samples and Molecular profiling studies. Illustrated is a diagram of the patient samples and molecular profiling studies used in the various analyses.

\section{A. Cuffdiff Chart}

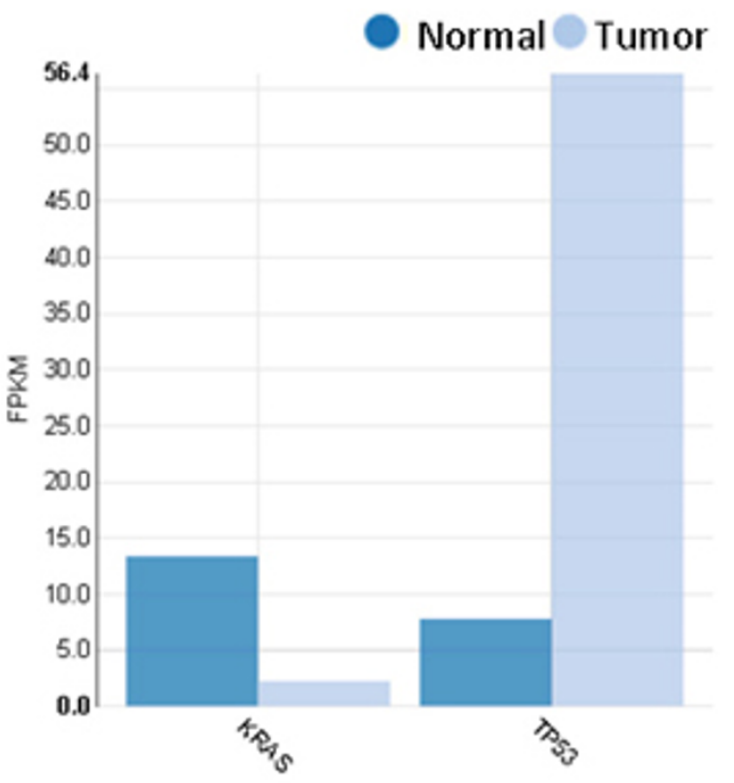

B.

\begin{tabular}{|l|l|l|l|r|r|r|r|r|}
\hline Gene & Locus & Sample 1 & Sample 2 & Value 1 & Value 2 & P value & Q value & Significant \\
\hline KRAS & $12: 25348149-25403870$ & Normal & Tumor & 13.3951 & $\mathbf{2 . 2 5 9 8 5}$ & $5.00 \mathrm{E}-05$ & $\mathbf{0 . 0 0 0 6 5 8 9}$ & Yes \\
\hline IPS3 & $17: 7565096-7606820$ & Normal & Tumor & $\mathbf{7 . 7 9 7 6 7}$ & $\mathbf{5 6 . 4 1 3 9}$ & $\mathbf{5 . 0 0 E - 0 5}$ & $\mathbf{0 . 0 0 0 6 5 8 9}$ & Yes \\
\hline
\end{tabular}

Figure 3 System Rendered Results for Key Cancer Biology Genes. Automatically rendered bar chart and table from a Cuffdiff dataset that was searched using two genes (KRAS and TP53), which have cancer biology significance in many cancers, including MM. The Cuffdiff Chart in subfigure $\mathbf{A}$ was quickly generated by a single point and click. All genes in the table (subfigure B) contain a significant p-value and q-value.

automatically generated by a single point and click. All genes in the table (subfigure B) contain a significant pvalue and q-value. Next, a triple integration was performed by combining the Cuffdiff gene data with WES and a microarray experiment. This is shown in Figure 4. Here the search criteria was based on a user defined five key gene group, which includes KRAS and TP53. The KRAS entry is circled for illustrative purposes. 


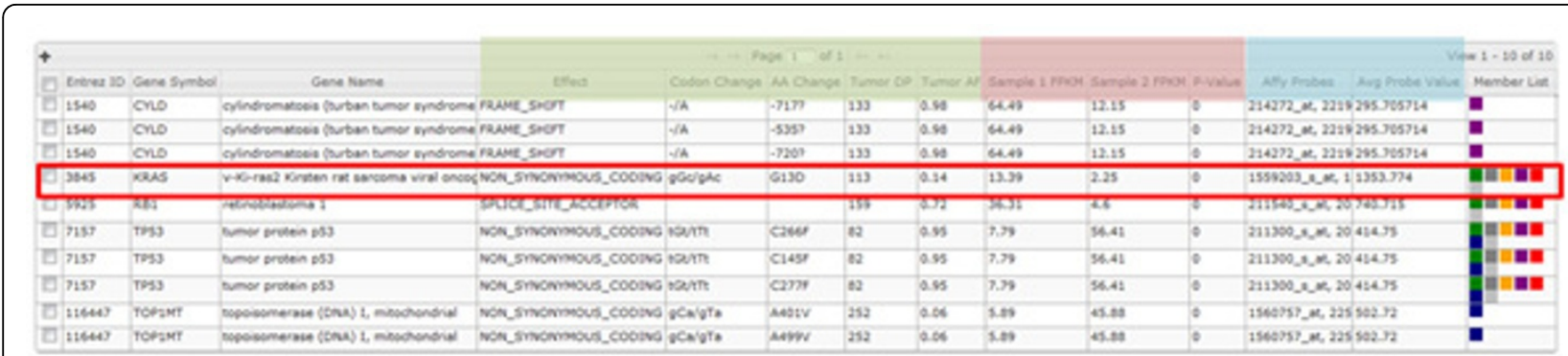

Figure 4 Triple Integration (WES, Cuffdiff Gene, Microarray). A triple integration was performed by combining Cuffdiff gene data with WES and a gene expression microarray experiment. The integrated WES data (green columns) includes: Effect, Codon Change, AA Change, Tumor DP (Depth), Tumor AF (Allelic Frequency). The corresponding RNA-seq data (light red columns), show the Sample 1 FPKM (pooled normal), Sample 2 FPKM (tumor), and p-value from Cufflinks. The microarray data (blue columns) display the corresponding Affy Probes, and Avg Probe Values. The grey columns are common across all modalities and show, Entrez ID, Gene Symbol, Gene Name, and Member List (gene list).

The integrated WES data (green columns) includes: Effect, Codon Change, AA Change, Tumor DP (Depth), Tumor AF (Allelic Frequency). Next, the corresponding RNA-seq data (light red columns) show the Sample 1 FPKM (pooled normal), Sample 2 FPKM (tumor), and $p$-value from Cufflinks. The microarray data (blue columns) display the corresponding Affy Probes, and Avg Probe Values. This minimal view shows KRAS with a non-synonymous hotspot mutation resulting in a change in the amino acid Glycine to Asparatic Acid at the $13^{\text {th }}$ position (G13D). This finding has a depth of coverage of 113 reads and an allelic frequency of $14 \%$. This is a known druggable mutation. The table in additional file 10 shows potential drug candidates through drug-gene interactions via custom automation/linkage (Figure 1, Subsystems Complementing Integrated Molecular Profiling Data Exploration, Drug-Gene interaction linkage) from our system to the DGIdb [24].

Additional file 11 shows a triple integration of data sets from WES, Cuffdiff isoforms, and gene expression microarray. There are two KRAS gene isoform transcripts from the Ensembl annotation with non-zero values in the column Sample 2 FPKM (tumor), namely ENST00000556131 and ENST00000311936. Figure 5, subfigure A shows an automated visualization of the FPKM Chart for KRAS and its associated isoforms. This was automatically generated by clicking the picture icon located in the rightmost region on the first line of the $K R A S$ entry, in subfigure B. In this view the novel isoform (i.e., CUFF.19733.1) was also fetched from the experimental data. The columns Transcript Id and $P$-Value show that only one transcript (ENST00000 311936) is significant. The visualization allows changes to be spotted quickly. A "hover over" feature, allows viewing the tumor or normal isoform FPKM values, in the ring/donut plot of subfigure A.

Further discovery and validation of the KRAS finding is illustrated in Figure 6. IGV automation is utilized, and by a single point and click on the web page entry for $K R A S$, relevant information is spontaneously sent to IGV. The transferred information includes data from Variant Analysis, RNA-seq aligned reads, WES aligned reads, the reference genome and RABT (Referenced Annotation Based Transcript) assembly. For illustrative purposes, the significant isoform, ENST00000311936, is circled in green, and the location of the non-synonymous hotspot mutation, G13D, is circled in red. A potential novel isoform (CUFF.19733.1) from the normal pool is circled in blue.

Further exploration, discovery, and validation is accomplished by using IGV to drive further into the integrated complex data sets, as is shown in additional file 12. The same circled entries and color assignments apply. IGV is used to validate the KRAS G13D hotspot mutation by examining the WES aligned reads. Since KRAS is known to code from the reverse strand, the point mutation is shown as a "T" on the IGV display. However, the RNAseq aligned reads do not appear to show evidence of the point mutation. This is confirmed by driving further into the IGV display and is shown in additional file 13. In this case, although there is a KRAS DNA point mutation indicating a non-synonymous G13D, it is not reflected into the transcriptome and therefore, will not be translated to protein. Since a mutated KRAS protein is the drug target, a planned therapeutic assignment, which is many times based solely on DNA gene mutational data, may now be reconsidered given the integrated transcriptome findings.

Additional file 14 shows IGV validation of a novel splice isoform (CUFF.19733.1). Although it is from the normal pool it is shown for illustrative purposes. The novel exon is circled in purple, and the isoform with closest similarity is ENST00000311936. Figure 7 shows automation utilizing $\mathrm{R}$ with the shiny package and Plot Protein [25] for the KRAS G13D hotspot mutation. A "lolliplot" is produced that demonstrates amino acid changes in the context of previously reported mutations and protein domains. The G13D mutation found in our experiment is colored red. 


\section{FPKM Chart for KRAS: 'v-Ki-ras2 Kirsten rat sarcoma viral oncogene homolog'}

A.

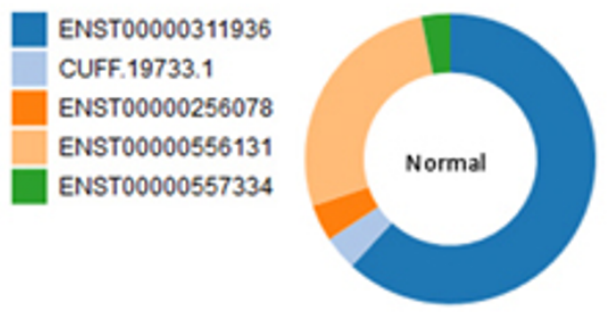

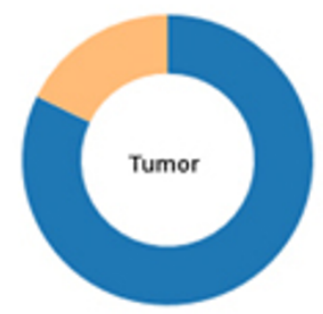

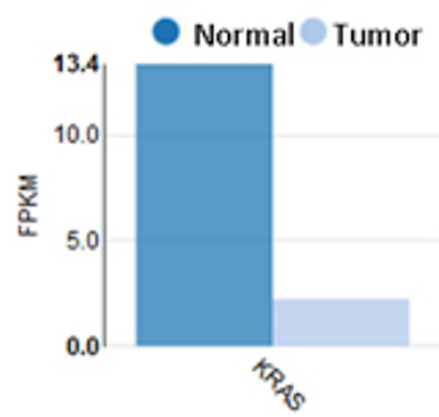

B.

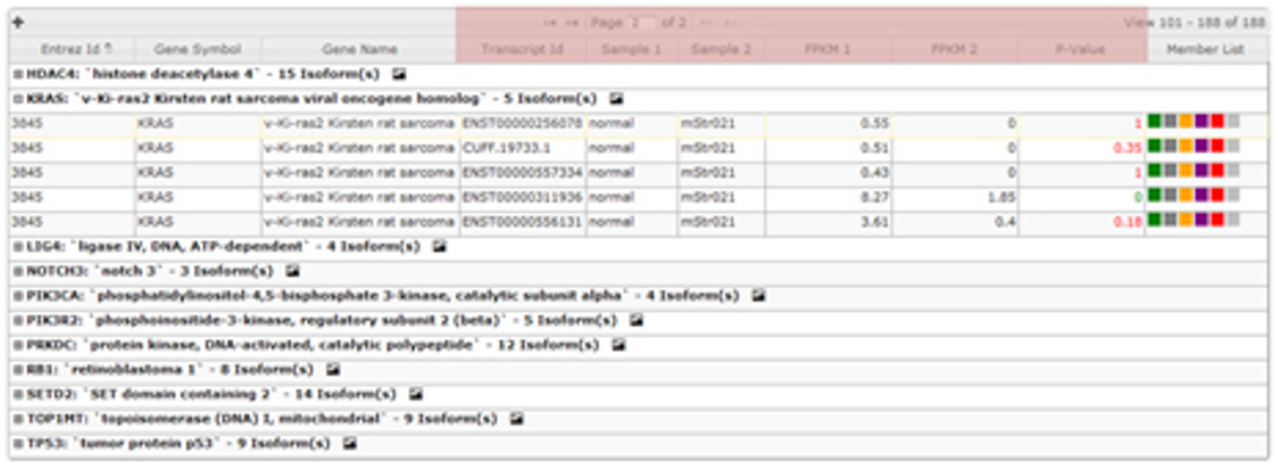

Figure 5 KRAS Automated Visualization and Exploration. Subfigure A shows an automated visualization of the FPKM Chart for KRAS and its associated isoforms. This was automatically generated by clicking the picture icon located in the rightmost region on the first line of the KRAS entry, in subfigure B. In this view, the novel isoform (i.e., CUFF.19733.1) was also fetched from the experimental data. The columns Transcript Id and P-Value show that only one transcript (ENST00000311936) is significant.

Other canonical mutations are colored blue. The RAS protein domain is colored green.

TP53 is a key tumor suppressor gene and known as the guardian of the genome. Cells that have a functional p53 pathway are less likely to transform. Genomic instability is a cardinal finding in p53 mutant cells. When TP53 is deleted in $M M$, a patient's likely survival becomes significantly shortened [26]. Classical tumor suppressor genes are usually characterized by nonsense or frame shift mutations that lead to truncated non-functional proteins. However, over $75 \%$ of all p53 mutations are missense and result in a single amino acid substitution. Accumulation of p53 mutants, to the point of showing a gain of function, can be seen because the mutant molecules can be more stable than the wild-type, and thus accrue [27-29].

Figure 3 shows the Cuffdiff Chart (subfigure A) and table (subfigure B) entries for TP53. Both the automatically generated bar chart and Value 2 (tumor) table entry clearly show TP53 is significantly overexpressed vs. normal. Additional file 15 shows a triple integration of experimental data sets from WES, Cuffdiff isoforms, and microarray. The red box is used to illustrate the seven TP53 transcript isoforms, which are associated with the appropriate non synonymous mutation (C277F or C266F or C145F) depending on how the exon with the point mutation $(\mathrm{G}>\mathrm{T})$ was spliced.

Approximately $90 \%$ of missense mutations for TP53 occur in the DNA binding domain, which spans amino acids 102-292, and all candidate SNVs in this experiment also fall in this region. The tumor depth is 82 reads and allelic frequency is $95 \%$. By a single click on any of the TP53 entries, we can jump directly into IGV, and begin to validate and further explore this SNV. This is illustrated in the figure found in additional file 16 . Since TP53 is coded on the reverse strand the point mutation appears as an "A" rather than "T", in IGV under the WES aligned reads section. In this case the RNA-seq aligned reads show concordance with the DNA findings. Together they provide stronger evidence for this biological finding. This important experimental data discovery was validated quickly due to the integrative and exploratory abilities of our system.

When TP53 is deleted, the effect and clinical ramifications are understood in MM and other cancers. However, the implications of TP53 missense mutations are not fully understood, and clearly may be important for therapeutic assignments and assessing prognosis. Thus, tools and approaches to track and study TP53 and other relevant 

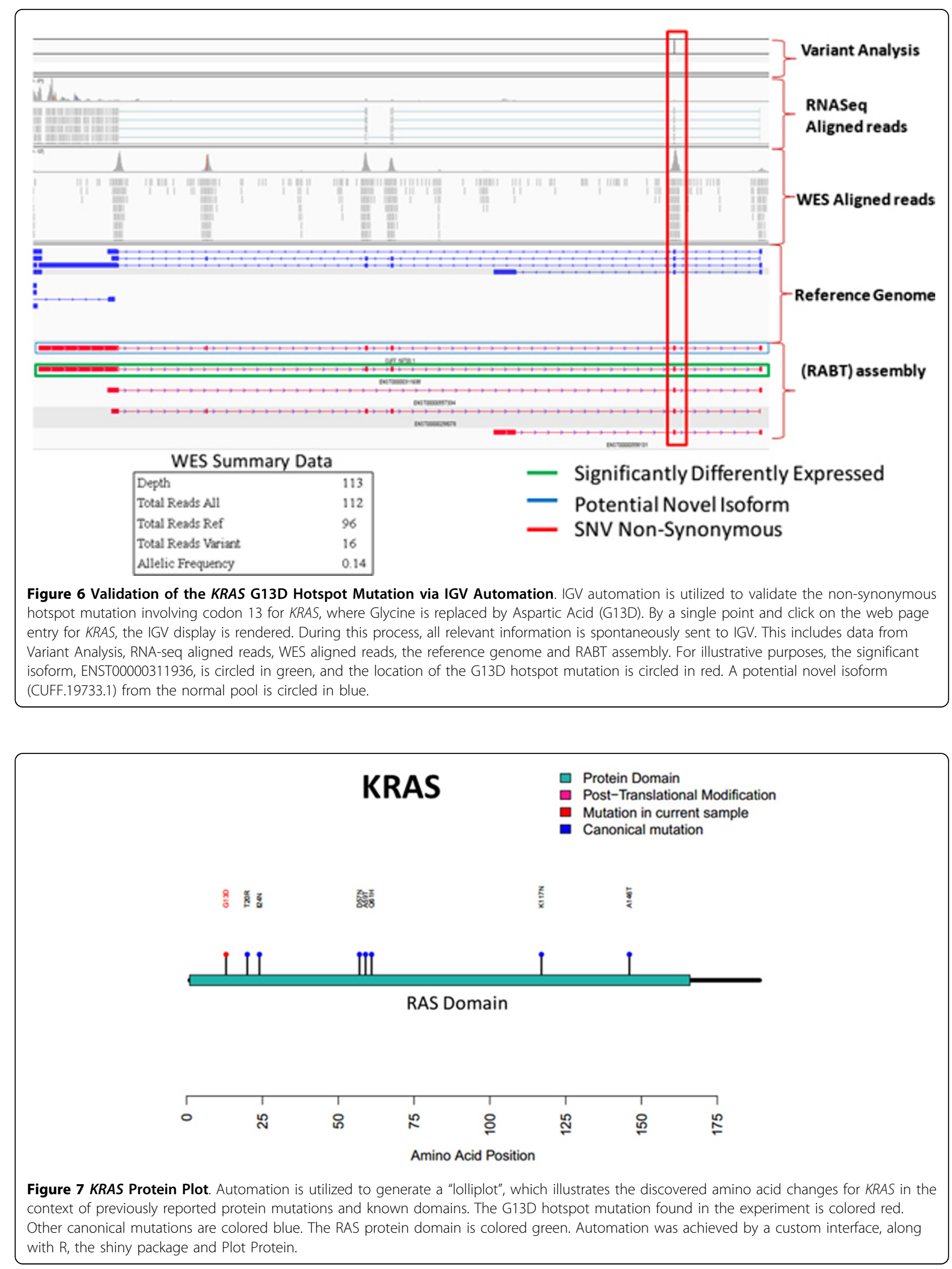
cancer mutations are needed. Our system provides a small step in this direction.

\section{Conclusions}

There is much to learn about the complexities of cancer genomes and NGS approaches are enabling a new understanding. As molecular profiling technologies evolve, it is critical to leverage what has been learned from historic techniques (e.g., microarrays) as new disruptive approaches (e.g., NGS) continue to gain a scientific presence and become a significant modality in clinical workflows (e.g., MySeqDx). These new and rapid changes are now reflected to the point that the science of computational biology is now driving many aspects of traditional "bench research". For instance, it is now common to use PCR to cross-validate specific computational findings. This is especially true in cancer. Furthermore, it is established that molecular heterogeneity results in complex genetic landscapes and are a common and confounding problem with many cancers. Demonstrated in this study are multi-modality integrative approaches, using KRAS and TP53 as examples, aimed to combat the heterogeneity dilemma, and bring more clarity to complex NGS studies.

The novel aspects of our approach are the abilities allowing for a rapid integration, exploration, discovery and validation of large and complex experimental data from a wide variety of NGS and microarray molecular profiling modalities, and are independent of any particular cancer type. Supported comparisons include: i) RNA-seq vs. Microarray, ii) WES vs. Microarray, iii) WES vs. RNA-seq, iv) WES vs. RNA-seq vs. Microarray (triple integration). The triple integration approach was illustrated in this study because it is the most difficult. To our knowledge, there is no existing software suite available, commercial or open source, which provides the triple integration feature. These abilities enable a more informed view of the intricate details present in the complex cancer biology, as was demonstrated with the KRAS and TP53 findings. The integrative KRAS findings indicate that the hotspot G13D DNA mutation does not appear to be translated into protein, thus negating the need for an expensive medicine targeting this particular mutation. TP53 integrative findings clearly displayed concordance between the DNA and RNA-seq transcriptome results and thus strengthen the biological evidence of the missense mutation.

There is a need to capture and model salient cancer biology knowledge gained from historic approaches. We continue to lay the groundwork for such endeavours concerning MM and other cancer types. It is our hope that such efforts will advance our understanding of the underlying cancer biology of MM, improve patient outcomes, and begin to further approach the concept of a cure.

\section{Additional material}

Additional file 1: Microarray vs. RNA-seq

Additional file 2: User Profile Management. User session profiles and custom gene groups may be created and modified enabling a more personalized experience with NGS Association System.

Additional file 3: Experiment Upload. A series of dialogs that profile the various steps for the creation of an example experimental study are shown. Once a patient is created, experimental details may be entered, and then the molecular profiling data can be scheduled for upload.

Additional file 4: Association Algorithm. Association tables and software procedures allow for the conversion between different gene identifiers. Conversions can be either direct or indirect depending on the starting and target IDs.

Additional file 5: Executive Console. The executive console provides basic study statistics as well as an overview of all imported and mapped study-related data. The ability to delete raw and/or mapped experimental data is provided from this interface.

Additional file 6: Patient Experiments View. All molecular profiling experiments for a patient can be viewed. A single modality or multiple modality session can be initiated.

Additional file 7: Filter Options. Filtering options help to quickly reduce thousands of results to targeted key genes of interest.

Additional file 8: Integrated Data Exploration, WES + Microarray (Min View). A minimal view of a WES and gene expression microarray integrated dataset. Grey colored columns are common across all modalities. Green columns are specific to WES and blue for microarray data. When present, RNA-seq columns are shaded light red.

Additional file 9: Integrated Data Exploration, WES + Microarray (Max View). A maximal view of a WES and gene expression microarray integrated dataset.

Additional file 10: Drug-Gene Interactions. These are acquired by linkage to DGIdb.

Additional file 11: Triple Integration (WES, Cuffdiff Isoform, Microarray). Displayed is a triple integration of data sets from WES, Cuffdiff isoforms, and gene expression microarray. Two KRAS gene isoform transcripts from the Ensembl annotation with non-zero values in the column Sample 2 FPKM (tumor), namely ENST00000556131 and ENST00000311936, are contained in the red circled region.

Additional file 12: Further Validation of the KRAS G13D Hotspot Mutation. IGV automation is utilized to further explore, discover, and validate the KRAS G13D hotspot mutation; by examining the WES aligned reads. Since KRAS is known to code from the reverse strand, the point mutation is shown as a "T" on the IGV display. The RNA-seq aligned reads do not appear to show evidence of the point mutation, and this finding requires further confirmation.

Additional file 13: Confirming a lack of transcription for the KRAS G13D Hotspot Mutation. IGV automation is again utilized to further explore and discover a lack of transcription for the KRAS G13D hotspot mutation. Although there is a KRAS DNA mutation, which is a known druggable target in many cancers including $M M$, there is no evidence of transcription. Therefore the mutational change will not be translated into protein. These integrative findings provide important information for clinical decision making and therapeutic assignments.

Additional file 14: Validating a KRAS Novel Splice Isoform. The KRAS novel splice isoform, CUFF.19733.1, was discovered in the NGS Association System, and is now further explored and validated by IGV automation. The novel exon is circled in purple and the isoform with the closest similarity is indicated.

Additional file 15: Triple Integration (WES, Cuffdiff Isoform, Microarray). A triple integrated view of experimental data sets from WES, Cuffdiff Isoforms, and microarray is shown. Circled in red are 
seven TP53 transcript isoforms associated with the nonsynonymous mutation (C277F or C266F or C145F) depending on how the exon with the point mutation ( $\mathrm{G}>\mathrm{T}$ ) was spliced.

Additional file 16: Validation of a TP53 Missense Mutation. RNA-seq aligned reads show concordance with the DNA findings (WES aligned reads) and are circled in red. Together they provide stronger evidence for the mutational result. This validation was accomplished by a single point and click.

\section{Competing interests}

The authors declare that they have no competing interests.

\section{Authors' contributions}

DJJ and MAB conceived and designed the study. MAB, SSC, EAP, CJH and DJJ performed the experiments and analyses. DJJ, MAB, SSC and EAP designed the software. MAB, SSC, and EAP implemented the software. DJJ and $M A B$ wrote the manuscript. All authors approved the manuscript.

\section{Acknowledgements}

Appreciation and thanks to Mr. Daniel Pollock for his programming assistance.

\section{Declarations}

Publication of this work was support by Program Project Grant CA 55813, from the National Institutes of Health $(\mathrm{NIH})$, National Cancer Institute (NCI), Bethesda, MD, USA.

This article has been published as part of BMC Bioinformatics Volume 15 Supplement 11, 2014: Proceedings of the 11th Annual MCBIOS Conference. The full contents of the supplement are available online at http://www. biomedcentral.com/bmcbioinformatics/supplements/15/S11.

Published: 21 October 2014

\section{References}

1. Johnsen JM, Nickerson DA, Reiner AP: Massively parallel sequencing: the new frontier of hematologic genomics. Blood 2013, 122(19):3268-3275

2. Collins FS, Hamburg MA: First FDA authorization for next-generation sequencer. N Engl J Med 2013, 369(25):2369-2371.

3. Kandoth C, McLellan MD, Vandin F, Ye K, Niu B, Lu C, Xie M, Zhang Q, McMichael JF, Wyczalkowski MA, et al: Mutational landscape and significance across 12 major cancer types. Nature 2013, 502(7471):333-339.

4. Mirnezami R, Nicholson J, Darzi A: Preparing for precision medicine. $\mathrm{N}$ Engl J Med 2012, 366(6):489-491.

5. Chapman MA, Lawrence MS, Keats JJ, Cibulskis K, Sougnez C, Schinzel AC, Harview CL, Brunet JP, Ahmann GJ, Adli M, et al: Initial genome sequencing and analysis of multiple myeloma. Nature 2011, 471(7339):467-472

6. Walker BA, Wardell CP, Melchor L, Hulkki S, Potter NE, Johnson DC, Fenwick K, Kozarewa I, Gonzalez D, Lord CJ, et al: Intraclonal heterogeneity and distinct molecular mechanisms characterize the development of $t$ $(4 ; 14)$ and $t(11 ; 14)$ myeloma. Blood 2012, 120(5):1077-1086.

7. Morgan GJ, Walker BA, Davies FE: The genetic architecture of multiple myeloma. Nature reviews Cancer 2012, 12(5):335-348.

8. Lohr JG, Stojanov P, Carter SL, Cruz-Gordillo P, Lawrence MS, Auclair D, Sougnez C, Knoechel B, Gould J, Saksena G, et al: Widespread genetic heterogeneity in multiple myeloma: implications for targeted therapy. Cancer cell 2014, 25(1):91-101.

9. Wang Z, Gerstein M, Snyder M: RNA-Seq: a revolutionary tool for transcriptomics. Nature reviews Genetics 2009, 10(1):57-63.

10. Chavan SS, Bauer MA, Peterson EA, Heuck CJ, Johann DJ Jr: Towards the integration, annotation and association of historical microarray experiments with RNA-seq. BMC bioinformatics 2013, 14(Suppl 14):S4

11. Shaughnessy JD, Zhan F, Burington BE, Huang Y, Colla S, Hanamura I, Stewart JP, Kordsmeier B, Randolph C, Williams DR, et al: A validated gene expression model of high-risk multiple myeloma is defined by deregulated expression of genes mapping to chromosome 1. Blood 2007, 109(6):2276-2284
12. Shaughnessy JD, Qu P, Usmani S, Heuck CJ, Zhang Q, Zhou Y, Tian E, Hanamura I, van Rhee F, Anaissie E, et al: Pharmacogenomics of bortezomib test-dosing identifies hyperexpression of proteasome genes, especially PSMD4, as novel high-risk feature in myeloma treated with Total Therapy 3. Blood 2011, 118(13):3512-3524.

13. Zhan F, Huang Y, Colla S, Stewart JP, Hanamura I, Gupta S, Epstein J, Yaccoby S, Sawyer J, Burington B, et al: The molecular classification of multiple myeloma. Blood 2006, 108(6):2020-2028.

14. Zhan F, Hardin J, Kordsmeier B, Bumm K, Zheng M, Tian E, Sanderson R, Yang $Y$, Wilson $C$, Zangari $M$, et al: Global gene expression profiling of multiple myeloma, monoclonal gammopathy of undetermined significance, and normal bone marrow plasma cells. Blood 2002, 99(5):1745-1757.

15. Davis S, Meltzer PS: GEOquery: a bridge between the Gene Expression Omnibus (GEO) and BioConductor. Bioinformatics 2007, 23(14):1846-1847.

16. Bolger AM, Lohse M, Usadel B: Trimmomatic: a flexible trimmer for Illumina sequence data. Bioinformatics 2014

17. Li H, Durbin R: Fast and accurate short read alignment with BurrowsWheeler transform. Bioinformatics 2009, 25(14):1754-1760.

18. Lunter G, Goodson M: Stampy: a statistical algorithm for sensitive and fast mapping of Illumina sequence reads. Genome Res 2011 21(6):936-939.

19. DePristo MA, Banks E, Poplin R, Garimella KV, Maguire JR, Hartl C, Philippakis AA, del Angel G, Rivas MA, Hanna M, et al: A framework for variation discovery and genotyping using next-generation DNA sequencing data. Nat Genet 2011, 43(5):491-498.

20. Saunders CT, Wong WS, Swamy S, Becq J, Murray $\amalg$, Cheetham RK: Strelka: accurate somatic small-variant calling from sequenced tumor-normal sample pairs. Bioinformatics 2012, 28(14):1811-1817.

21. Cingolani P, Platts A, Wang le L, Coon M, Nguyen T, Wang L, Land SJ, Lu X, Ruden DM: A program for annotating and predicting the effects of single nucleotide polymorphisms, SnpEff: SNPs in the genome of Drosophila melanogaster strain w1118; iso-2; iso-3. Fly (Austin) 2012 6(2):80-92

22. Thorvaldsdottir H, Robinson JT, Mesirov JP: Integrative Genomics Viewer (IGV): high-performance genomics data visualization and exploration. Briefings in bioinformatics 2013, 14(2):178-192.

23. Lee HC, Shah JJ, Orlowski RZ: Novel approaches to treatment of doublerefractory multiple myeloma. American Society of Clinical Oncology educational book / ASCO American Society of Clinical Oncology Meeting 2013.

24. Griffith M, Griffith OL, Coffman AC, Weible JV, McMichael JF, Spies NC, Koval J, Das I, Callaway MB, Eldred JM, et al: DGldb: mining the druggable genome. Nature methods 2013, 10(12):1209-1210.

25. Turner T: Plot protein: visualization of mutations. Journal of clinical bioinformatics 2013, 3(1):14.

26. Drach J, Ackermann J, Fritz E, Kromer E, Schuster R, Gisslinger $H_{\text {, }}$ DeSantis M, Zojer N, Fiegl M, Roka S, et al: Presence of a p53 gene deletion in patients with multiple myeloma predicts for short survival after conventional-dose chemotherapy. Blood 1998, 92(3):802-809.

27. Goh $A M$, Coffill CR, Lane DP: The role of mutant p53 in human cancer. J Pathol 2011, 223(2):116-126.

28. Goldstein I, Marcel V, Olivier M, Oren M, Rotter V, Hainaut P: Understanding wild-type and mutant p53 activities in human cancer: new landmarks on the way to targeted therapies. Cancer Gene Ther 2011, 18(1):2-11.

29. Brown CJ, Lain S, Verma CS, Fersht AR, Lane DP: Awakening guardian angels: drugging the p53 pathway. Nature reviews Cancer 2009, 9(12):862-873

\section{doi:10.1186/1471-2105-15-S11-S3}

Cite this article as: Bauer et al:: Leveraging the new with the old: providing a framework for the integration of historic microarray studies with next generation sequencing. BMC Bioinformatics 2014 15(Suppl 11):S3. 